\title{
Estudio de las variables pedagógicas en tareas de entrenamiento en fútbol-base según el mesociclo competitivo. Un estudio de casos \\ Study of the pedagogical variables in grassroots football training tasks by competitive mesocycle. A case study \\ José Martín Gamonales-Puerto, Carlos David Gómez-Carmona, Kiko León, Jesús Muñoz-Jiménez, Sergio José Ibáñez \\ Universidad de Estremadura (España)
}

Resumen. El objetivo del presente estudio fue conocer las tareas diseñadas por un entrenador de fútbol-base, así como analizar las diferencias entre la variable Tipo de Mesociclo (TMe) y las variables pedagógicas que influyen en el diseño de tareas llevadas a cabo durante dos mesociclos competitivos en categoría sub-18. Se analizaron un total de 89 tareas repartidas en 14 sesiones durante dos meses, febrero $(n=8)$ y marzo $(n=6)$. El instrumento empleado para el análisis de las tareas fue el Sistema Integral para el Análisis de las Tareas de Entrenamiento (SIATE), donde se analizaron las siguientes variables pedagógicas: Situación de juego (SJ), Presencia de portero (POR), Fase de juego (FJ), Tipo de contenido (CONT-G), Medio de iniciación deportiva (MIE), Nivel de oposición (NO) y Línea de juego (LJ). Los resultados muestran una progresión en la complejidad del diseño de las tareas de entrenamiento. Además, existen diferencias en el diseño de tareas entre los mesociclos analizados, POR y $L J$, con un grado de asociación bajo. Es fundamental diseñar entrenamientos donde se trabajen diferentes líneas de juego. Por tanto, el SIATE ha demostrado ser una herramienta óptima para registrar y analizar las tareas en fútbol-base.

Palabras claves: fútbol, SIATE, tareas, entrenador.

\begin{abstract}
The aim of the present study was to assess the tasks designed by a youth football team coach, as well as to analyze the differences between the independent variable Type of Mesocycle (TMe) and the dependent pedagogical variables influencing the design of training tasks performed during a two-months competitive period in the U-18 category. A total of 89 tasks, distributed in 14 sessions between February $(n=8)$ and march $(n=6)$ were analyzed. The tool utilized for task analysis was the Integral System to Training Tasks Analysis (SIATE, in Spanish), where the following pedagogical variables were registered: Playing situation (SJ), Goalkeeper presence (POR), Playing phase (FJ), Type of content (CONT-G), Sport initiation means (MIE), Opposition level (NO) and Playing role (LJ). The results showed a progression in the complexity of the designed training tasks, finding differences between the analyzed mesocycles. The variables $P O R$ and $L J$ obtained a low grade of association. Designing trainings where different lines of play are worked is essential. Therefore, the SIATE has shown to be an optimal tool for registering and analyzing training tasks in grassroots football.
\end{abstract}

Key words: Football, SIATE, tasks, coach.

\section{Introducción}

Actualmente, existe la necesidad de describir y explicar la realidad del proceso de entrenamiento y competición para poder incrementar las posibilidades de rendimiento en competición (Lago, 2008). El entrenamiento asume un importante papel en la adquisición y perfeccionamiento de las habilidades técnico-tácticas (Silva, Sánchez-Bañuelos, Gargante \& Anguera, 2005). Siendo, las tareas de entrenamiento que diseñan los técnicos deportivos las unidades más concretas del proceso de planificación y programación del entrenamiento (Sáenz-López, Feu \& Ibáñez, 2006), las cuales permiten desarrollar los objetivos planificados a través de la práctica de los contenidos deportivos (Ibáñez, Feu \& Cañadas, 2016). Además, la experiencia de los formadores juega un papel clave en el diseño, selección y evolución de las tareas diseñadas durante los entrenamientos (Clemente, Martins \& Mendes, 2015). Por ello, es necesario implementar de forma implícita y explicita diferentes concepciones metodológicas durante los entrenamientos (Ibáñez, Feu \& Cañadas, 2016).

Los métodos de enseñanza-aprendizaje más utilizadas en la práctica deportiva son: los modelos tradicionalesmecanicistas, y los modelos constructivistas-alternativos (Alarcón, Cárdenas, Miranda, Ureña \& Pilar, 2010). En los deportes de invasión, cada vez es más frecuente utilizar

Fecha recepción: 07-03-19. Fecha de aceptación: 12-06-19

José Martín Gamonales-Puerto

josemartingamonales@gmail.com metodologías alternativas basadas en ideas constructivistas que buscan la implicación cognitiva y la compresión del juego (Kirk \& MacPhail, 2002), que influye en el diseño de las situaciones durante la práctica deportiva (Cañadas, Ibáñez, García-Rubio \& Sáez, 2010; Cañadas, Ibáñez, Feu, GarcíaRubio \& Parejo, 2011). Por ello, la selección de una metodología repercute indirectamente en el diseño y selección de las tareas de entrenamiento, y por consiguiente en el proceso formativo del deportista (Mancha-Triguero, GarcíaCeberino, Antúnez \& García-Rubio, 2018).

Durante el proceso de entrenamiento, los formadores manipulan diferentes variables para el diseño de las tareas (Gracia, García-Rubio, Cañadas \& Ibáñez, 2014), independientemente del posicionamiento metodológico y su perfil con la intervención práctica real (Ibáñez et al., 2016). En función de la metodología empleada, las demandas que presente el entrenamiento se verán afectadas (González-Espinosa, Ibáñez, Feu y Galatti, 2017), lo cual implica una diferente relación con respecto a la carga de competición (Sánchez, 2007), definida como la suma de estímulos a los que se enfrenta el deportista durante un periodo concreto de tiempo (Coque, 2008). Esta carga de entrenamiento carece de control y seguimiento como consecuencia del alto coste económico de los dispositivos de medida para la valoración del esfuerzo(Del Campo, Álvarez \& Lorenzo, 2008). No todos los equipos profesionales, y en especial los equipos en formación, poseen de tecnología para controlar la carga de entrenamiento, a través de tecnología de Sistema de Posicionamiento Global (GPS) (Bastida- 
Castillo, Gómez-Carmona, de la Cruz Sánchez \& Pino-Ortega, 2019; Malone, Lovell, Varley \& Coutts, 2017), o mediante tecnología de Radiofrecuencia de Ultra-banda Ancha (UWB) (Bastida-Castillo et al., 2019; Luteberget, Spencer \& Gilgien, 2018). El control en el deporte base puede realizarse de diferente modo, y siempre dependiendo del instrumental (Mancha-Triguero et al., 2018), así como de los recursos económicos disponibles. Para el registro y análisis de las tareas de entrenamiento en el deporte base, se han creado diferentes herramientas objetivas con la finalidad de categorizar las tareas, y entre las que se encuentra el instrumento denominado Sistema Integral para el Análisis de las Tareas de Entrenamiento (SIATE) (Ibañez et al. 2016).

En la literatura científica, existen diferentes estudios que emplean la herramienta SIATE. Concretamente en baloncesto, los diferentes trabajos permiten conocer las situaciones de juego empleadas (Cañadas, Ibáñez, García-Rubio, Parejo \& Feu, 2011; Cañadas, Ibáñez, García, Parejo \& Feu, 2013), los contenidos de entrenamiento (Cañadas \& Ibáñez, 2010; Cañadas, Ibáñez \& Leite, 2015), los medios de entrenamiento (Cañadas, Ibáñez, Feu, García-Rubio \& Parejo, 2011; Cañadas, Parejo, Ibáñez, García-Rubio \& Feu, 2009), las fases de juego (Cañadas, Ibáñez, García-Rubio, Parejo \& Feu, 2012; Mancha-Triguero et al., 2018), la relación entre las variables pedagógicas del entrenamiento (Cañadas, Rodríguez, Feu, Parejo \& García-Rubio, 2013), la carga durante una tarea (Gracia, García-Rubio, Cañadas \& Ibáñez, 2014; Reina, ManchaTriguero, Feu \& Ibáñez, 2017), e incluso la metodología empleada (González-Espinosa, Ibáñez \& Feu, 2017) a lo largo de la temporada. En balonmano, los trabajos existentes permiten conocer el tipo de participación y las líneas de juego en relación a los contenidos desarrollados durante el proceso de entrenamiento (Dallegrave, Mendes \& Vieira do Nascimento, 2018), y la carga externa (García-Ceberino, Gamero, González-Espinosa, García-Rubio \& Feu, 2018). En fútbol-base, también se ha utilizado la herramienta SIATE, permitiendo conocer los medios de iniciación al entrenamiento, así como las relaciones con las variables pedagógicas que influyen en el diseño de las tareas durante un mesociclo (Gómez-Carmona, García-Rubio, Muñoz-Jiménez \& Gamonales, 2018), e incluso, diferencias entre equipos y entrenadores con diferentes experiencia y formación (Gamonales, Gómez-Carmona, León, García-Santos, Gamero-Portillo \& Muñoz-Jiménez, 2019).

A partir de la bibliográfica consultada, existe la necesidad de ampliar el conocimiento, así como conocer las características de las tareas de entrenamiento en fútbol-base. Por ello, los objetivos de este estudio fueron: a) conocer las tareas diseñadas por un entrenador de fútbol-base, sub-18, y b) analizar las diferencias entre la variable Tipo de Mesociclo (TMe) y las variables pedagógicas que influyen en el diseño de tareas.

\section{Método}

\section{Diseño}

Este estudio se encuadra dentro de una estrategia descriptiva de tipo selectivo (Ato, López \& Benavente, 2013), con la finalidad de conocer la planificación y selección de tareas de los entrenadores en fútbol-base.

\section{Muestra}

Se analizaron todas las sesiones de entrenamiento $(n=14)$ correspondientes a dos mesociclos; competitivo VI $(n=8)$ y competitivo VII $(n=6)$, diseñados y ejecutados por el entrenador de fútbol-base de un club de categoría sub-18 de primera división regional, dando lugar a un total de 89 tareas. En la tabla 1, se muestra las principales características de los mesociclos analizados.

\begin{tabular}{|c|c|c|c|c|}
\hline \multirow[b]{2}{*}{ Mesociclos } & \multirow[b]{2}{*}{ Tareas } & \multicolumn{3}{|c|}{ Tipos de tiempo } \\
\hline & & Tiempo total & $\begin{array}{c}\text { Tiempo } \\
\text { explicación }\end{array}$ & Tiempo útil \\
\hline \multirow{4}{*}{ Competitivo VI 47} & Media \pm DE & $734.04 \pm 644.38$ & $58.51 \pm 97.40$ & $675.53 \pm 571.55$ \\
\hline & 47 Máximo & 3360 & 600 & 2760 \\
\hline & Mínimo & 180 & 0 & 160 \\
\hline & Media $\pm \mathrm{DE}$ & $624.52 \pm 573.72$ & $37.62 \pm 38.67$ & $586.90 \pm 571.18$ \\
\hline \multirow{2}{*}{ Competitivo VII 4} & 42 Máximo & 3120 & 120 & 3120 \\
\hline & Mínimo & 140 & 0 & 120 \\
\hline \multirow{3}{*}{ Total casos } & Media $\pm \mathrm{DE}$ & $682.36 \pm 611.08$ & $48.65 \pm 75.94$ & $633.71 \pm 569.85$ \\
\hline & 89 Máximo & 3360 & 600 & 3120 \\
\hline & Mínimo & 140 & 0 & 120 \\
\hline
\end{tabular}

\section{Instrumento y variables analizadas}

Se registraron todas las tareas mediante la herramienta SIATE (Ibañez et al. 2016), analizándose las variables pedagógicas: Situación de juego (SJ), Presencia de portero (POR), Fase de juego (FJ), Tipo de contenido (CONT-G), Medio de iniciación deportiva (MIE), Nivel de oposición (NO) y Línea de juego (LJ).

- Situación de Juego (SJ): Agrupaciones de jugadores que los entrenadores diseñan para cada una de las tareas, pudiendo ser cambiantes o fijas durante la ejecución. En el mismo se incrementa la complejidad desde la situación de $1 \mathrm{vs} 0$ hasta $11 \mathrm{vs} 11$.

- Presencia de Portero (POR): Indica si existe guardameta durante la tarea o por el contrario se prescinde del rol en las agrupaciones.

- Fase de Juego (FJ): Esta determinada por la posesión del balón y se divide en rol definido durante la tarea (ataque o defensa) o fase mixta (ataque-defensa). Si no existe el aspecto competitivo, la fase de juego se clasifica en calentamiento, preparación física, recuperación o actividades de evaluación.

- Tipo de Contenido (CONT-G): Determina el contenido a trabajar durante la sesión de entrenamiento, siguiendo a Ibáñez (2002) se agrupan en contenidos individuales, grupales y de equipo, tanto para el desarrollo en fase de ataque como defensa, así como para la conductas tácticas y gestos técnicos.

- Medio de Iniciación Deportiva (MIE): Representa las actividades motrices deportivas que sirven para el desarrollo de los contenidos técnico-tácticos, desde la menor complejidad (ejercicio de aplicación simple) hasta la mayor complejidad (deporte y competición).

- Nivel de Oposición (NO): Identifica el tipo de oponente que se interpone entre el/los ejecutantes y conseguir el objetivo de la tarea. Esta se clasifica en: sin oposición, obstáculos estáticos, obstáculos dinámicos, oposición modulada y oposición.

\section{Procedimiento}

El entrenador planificó y diseñó las distintas tareas a llevar a cabo durante los dos mesociclos competitivos. Una vez recopiladas las tareas, dos evaluadores externos, previamente entrenados, categorizaron las tareas en base al instru- 
mento previamente descrito. Se comprobó la fiabilidad intercodificador mediante el índice Multirater Kappa Free (Randolph, 2005), que asume que un valor de 0.70 o superior indica un alto grado de acuerdo entre codificadores. El proceso aplicado fue similar a los existentes en la literatura científica en otros contextos deportivos como en rugby (Villarejo, Ortega, Gómez \& Palao, 2014), o en fútbol a 5 (Gamonales, Muñoz-Jiménez, León-Guzmán \& Ibáñez, 2018). El resultado de fiabilidad inter-codificador fue como mínimo de 0.89 en todas las variables. Posteriormente, los observadores registraron todas las tareas de entrenamiento $(n=89)$ de los dos mesociclos de forma individual, para después tratar los datos.

\section{Análisis estadístico}

Se realizó un análisis descriptivo de todas las variables. Para analizar las diferencias existentes entre los diferentes mesociclos (variable, TMe) de tareas de entrenamiento dise-

\begin{tabular}{|c|c|c|c|c|}
\hline \multirow{3}{*}{$\begin{array}{c}\text { Variables } \\
\text { Situaciones de juego (SJ) }\end{array}$} & \multicolumn{4}{|c|}{ Tipo de mesociclo } \\
\hline & \multicolumn{2}{|c|}{ Competitivo VI } & \multicolumn{2}{|c|}{ Competitivo VII } \\
\hline & $n$ & $\%$ & $n$ & $\%$ \\
\hline $1 \times 0$ & 4 & 8.5 & 3 & 7.1 \\
\hline $1 \times 1$ & 1 & 2.1 & 1 & 2.4 \\
\hline $2 \times 0$ & 0 & 0.0 & 1 & 2.4 \\
\hline $2 \times 2$ & 0 & 0.0 & 1 & 2.4 \\
\hline $2 \times 3$ & 0 & 0.0 & 1 & 2.4 \\
\hline $3 \times 0$ & 2 & 4.3 & 0 & 0.0 \\
\hline $3 \times 1$ & 0 & 0.0 & 0 & 0.0 \\
\hline $3 \times 2$ & 0 & 0.0 & 0 & 0.0 \\
\hline $3 \times 3$ & 3 & 6.4 & 1 & 2.4 \\
\hline $4 \times 0$ & 5 & 10.6 & 1 & 2.4 \\
\hline $4 \times 2$ & 1 & 2.1 & 0 & 0.0 \\
\hline $4 \times 3$ & 0 & 0.0 & 1 & 2.4 \\
\hline $5 \times 1$ & 0 & 0.0 & 0 & 0.0 \\
\hline $5 \times 2$ & 0 & 0.0 & 0 & 0.0 \\
\hline $5 \times 3$ & 0 & 0.0 & 0 & 0.0 \\
\hline $5 \times 5$ & 1 & 2.1 & 1 & 2.4 \\
\hline $6 \times 6$ & 0 & 0.0 & 2 & 4.8 \\
\hline $7 \times 1$ & 0 & 0.0 & 0 & 0.0 \\
\hline $7 \times 2$ & 0 & 0.0 & 0 & 0.0 \\
\hline $7 \times 7$ & 0 & 0.0 & 2 & 4.8 \\
\hline $8 \times 7$ & 10 & 21.3 & 8 & 19.0 \\
\hline $8 \times 8$ & 1 & 2.1 & 0 & 0.0 \\
\hline $9 \times 1$ & 1 & 2.1 & 0 & 0.0 \\
\hline $9 \times 9$ & 0 & 0.0 & 0 & 0.0 \\
\hline $10 \times 10$ & 0 & 0.0 & 1 & 2.4 \\
\hline $\mathrm{N} \times \mathrm{N}$ & 6 & 12.8 & 4 & 9.5 \\
\hline Combinado & 10 & 21.3 & 14 & 33.3 \\
\hline Presencia de portero (POR) & $n$ & $\%$ & $n$ & $\%$ \\
\hline Con portero & 18 & 38.3 & 9 & 21,4 \\
\hline Sin portero & 29 & 61.7 & 33 & 78,6 \\
\hline Fase de juego (FJ) & $n$ & $\%$ & $n$ & $\%$ \\
\hline Ataque & 7 & 14.9 & 4 & 9.5 \\
\hline Defensa & 4 & 8.5 & 0 & 0.0 \\
\hline Mixta & 23 & 48.9 & 21 & 50.0 \\
\hline Calentamiento & 2 & 4.3 & 0 & 0.0 \\
\hline Preparación física & 11 & 23.4 & 17 & 40.5 \\
\hline Tipo de contenido (CONT-G) & $n$ & $\%$ & $n$ & $\%$ \\
\hline CTTGA & 0 & 0.0 & 1 & 2.4 \\
\hline CTTCA & 27 & 57.4 & 20 & 47.6 \\
\hline CTTIA & 0 & 0.0 & 1 & 2.4 \\
\hline GTTIA & 6 & 12.8 & 4 & 9.5 \\
\hline Pase & 0 & 0.0 & 0 & 0.0 \\
\hline Calentamiento & 5 & 10.6 & 8 & 19.0 \\
\hline Preparación física & 5 & 10.6 & 6 & 14.3 \\
\hline Partido entrenamiento & 4 & 8.5 & 2 & 4.8 \\
\hline Medio de iniciación al entrenamiento (MIE) & $n$ & $\%$ & $n$ & $\%$ \\
\hline Ejercicio de aplicación simple & 8 & 17.0 & 7 & 16.7 \\
\hline Ejercicio de aplicación compleja & 4 & 8.5 & 5 & 11.9 \\
\hline Juego simple específico & 0 & 0.0 & 0 & 0.0 \\
\hline Juego complejo inespecífico & 0 & 0.0 & 1 & 2.4 \\
\hline Juego complejo específico & 23 & 48.9 & 22 & 52.4 \\
\hline Pre-deporte / deporte adaptado & 3 & 6.4 & 1 & 2.4 \\
\hline Deporte & 5 & 10.6 & 4 & 9.5 \\
\hline Competición & 4 & 8.5 & 2 & 4.8 \\
\hline Línea de juego / grupo de jugadores (LJ) & $n$ & $\%$ & $n$ & $\%$ \\
\hline Portero & 4 & 8.5 & 0 & 0.0 \\
\hline Lateral & 0 & 0.0 & 0 & 0.0 \\
\hline Extremo & 5 & 10.6 & 1 & 2.4 \\
\hline Delantero & 1 & 2.1 & 1 & 2.4 \\
\hline Equipo & 37 & 78.7 & 40 & 95.2 \\
\hline Nivel de oposición (NO) & $n$ & $\%$ & & \\
\hline $\begin{array}{l}\text { Sin oposición } \\
\end{array}$ & 21 & 44.7 & 18 & 42.9 \\
\hline Con obstáculos dinámicos & 0 & 0.0 & 2 & 4.8 \\
\hline Con oposición & 26 & 55.3 & 22 & 52.4 \\
\hline
\end{tabular}

ñados por el técnico deportivo, se empleó Chi cuadrado $(\div 2)$ y Coeficiente Phi (öc) de Cramer (Newell, Aitchison \& Grant, 2014). El nivel de asociación del indicador öc de Cramer se interpretó a través de la propuesta de Crewson (2006). El grado de asociación entre las variables dependientes del estudio (variables pedagógicas) con la variable independiente del estudio (TMe) se realizó a través de los Residuos Tipificados Corregidos (RTC) de las tablas de contingencia (Field, 2009).

\section{Resultados}

En la tabla 2, se muestran los resultados del análisis descriptivo de las variables pedagógicas. Los resultados muestran la utilización de una amplia variedad de variables pedagógicas, siendo las más utilizadas la Situación de Juego 8x7 (VI: 21.3\%; VII: 19.0\%) y combinado(VI: 21.3\%; VII: 33.3\%), sin Presencia de Portero (VI: 61.7\%; VII: 78.6\%), en Fase de Juego mixta (VI: 48.9\%; VII: 50.0\%) y preparación física (VI: 23.4\%; VII: 40.5\%), con Tipo de Contenido relacionado con Conductas Técnico-Tácticas de Ataque (VI: 57.4\%; VII: 47.6\%), con juegos complejos específicos en relación al Medio de Iniciación al Entrenamiento (VI: 48.9\%; VII: 52.4\%), trabajando la Línea de Juego equipo (VI: 78.7\%; VII: 95.2\%) y combinando sin oposición (VI: 44.7\%; VII: 42.9\%)y con oposición (VI: 55.3\%; VII: 52.4\%) en cuanto al Nivel de Oposición.

En la tabla 3, se muestran las relaciones entre las variables pedagógicas y la variable TMe. Los resultados de las diferencias entre los mesociclos diseñados muestran que existen asociaciones significativas en las variables de estudio Presencia de Portero $(\div 2=6.99 ; p=0.03 ; \ddot{o c}=0.22$ asociación baja) y Línea de Juego $(\div 2=7.52 ; p=0.04 ; \ddot{c} c=0.27$ asociación baja) en función de TMe. Para analizar el grado de asociación se muestran los Residuos Tipificados Corregidos (RTC) (Tabla 4). Respecto a la variable Presencia de Portero está nos muestra una mayor probabilidad de tareas

\begin{tabular}{|c|c|c|c|c|c|c|}
\hline \multirow{2}{*}{ Variables pedagógicas } & \multicolumn{6}{|c|}{$\begin{array}{c}\text { Tipo de } \\
\text { mesociclo }\end{array}$} \\
\hline & ?2 & gl. & Sig. & $f c$ & Sig. & $\begin{array}{c}\text { Nivel de } \\
\text { asociación }\end{array}$ \\
\hline Situaciones de juego (SJ) & 20.883 & 19 & 0.343 & 0.484 & 0.343 & -- \\
\hline Presencia de portero (POR) & 6.992 & 1 & $0.031^{*}$ & 0.220 & 0.031 & Baja \\
\hline Fase de juego (FJ) & 7.939 & 4 & 0.094 & 0.299 & 0.094 & -- \\
\hline Tipo de contenido (CONT-G) & 6.231 & 7 & 0.513 & 0.265 & 0.513 & \multirow{2}{*}{$\begin{array}{l}-- \\
--\end{array}$} \\
\hline Medio al entrenamiento (MIE) & 2.705 & 6 & 0.845 & 0.174 & 0.845 & \\
\hline Línea de juego (LJ) & 7.523 & 3 & $0.048^{*}$ & 0.271 & 0.048 & Baja \\
\hline Nivel de Oposición (NO) & 2.290 & 2 & 0.318 & 0.160 & 0.318 & - \\
\hline \multicolumn{7}{|c|}{$\begin{array}{l}\text { Tabla } 4 . \\
\text { Nivel de contingencia con los RTC. }\end{array}$} \\
\hline \multirow{2}{*}{ Variables } & & & \multicolumn{4}{|c|}{ Tipo de mesociclo } \\
\hline & & & Compet & itivo VI & Competitivo Vl & Total \\
\hline \multicolumn{7}{|l|}{ Presencia de portero (POR) } \\
\hline Con portero & \multicolumn{2}{|l|}{$\begin{array}{c}n \\
R T C\end{array}$} & \multicolumn{2}{|c|}{$\begin{array}{c}18 \\
2.0^{*}\end{array}$} & $\begin{array}{c}9 \\
-2.0^{*} \\
\end{array}$ & 27 \\
\hline Sin portero & \multicolumn{2}{|l|}{$\begin{array}{c}n \\
R T C\end{array}$} & \multicolumn{2}{|c|}{$\begin{array}{c}29 \\
-2.2 *\end{array}$} & $\begin{array}{c}33 \\
2.2^{*}\end{array}$ & 62 \\
\hline Total & $n$ & & & & 42 & 89 \\
\hline \multicolumn{7}{|l|}{ Línea de juego (LJ) } \\
\hline Portero & \multicolumn{2}{|l|}{$\begin{array}{c}n \\
R T C\end{array}$} & \multicolumn{2}{|c|}{$\begin{array}{c}4 \\
2.0^{*}\end{array}$} & $\begin{array}{c}0 \\
-2.0^{*}\end{array}$ & 4 \\
\hline Lateral & $\begin{array}{c}n \\
R T C\end{array}$ & & \multicolumn{2}{|c|}{$\begin{array}{c}5 \\
2.3 *\end{array}$} & $\begin{array}{c}1 \\
-2.3^{*}\end{array}$ & 6 \\
\hline Extremo & $\begin{array}{c}n \\
R T C\end{array}$ & & \multicolumn{2}{|c|}{$\begin{array}{c}1 \\
-0.1 \\
\end{array}$} & $\begin{array}{c}1 \\
0.1\end{array}$ & 2 \\
\hline Delantero & $\begin{array}{c}n \\
R T C\end{array}$ & & & & $\begin{array}{c}1 \\
0.1 \\
\end{array}$ & 2 \\
\hline Equipo & $\begin{array}{c}n \\
\text { RTC }\end{array}$ & & & & $\begin{array}{c}40 \\
2.9 * \\
\end{array}$ & 77 \\
\hline Total & $n$ & & & & 42 & 89 \\
\hline
\end{tabular}


con portero en el Competitivo VI $(R T C=2.0)$ respecto al Competitivo VII donde hay una mayor probabilidad de diseñar tareas sin portero $(R T C=2.2)$. Finalmente, en la variable Línea de Juego existe una mayor probabilidad de realizar tareas específicas para portero $(R T C=2.0)$ y laterales $(R T C=$ 2.3) en el Competitivo VI, mientras que en el Competitivo VII existe una mayor probabilidad de realiza tareas donde participe todo el equipo $(R T C=2.9)$.

\section{Discusión}

Los objetivos de la investigación fueron identificar las tareas diseñadas por un entrenador de fútbol-base, sub-18, así como estudiar las diferencias existentes en el diseño de las tareas de entrenamiento entre los dos mesociclos competitivos, con la finalidad de ampliar el conocimiento científico relacionado con el fútbol de formación. Los resultados obtenidos muestran que el técnico deportivo especialista en fútbol utilizó una gran variedad de variables pedagógicas para diseñar sus tareas de entrenamiento en categoría juvenil. Principalmente, empleó situaciones de juego (SJ) de $8 x 7$ y combinadas, sin portero, donde todo el equipo trabajaba de forma mixta (transiciones de ataque-defensa), los contenidos calentamiento, preparación física, CTTCA y partido entrenamiento mediante juegos complejos específicos y ejercicios de aplicación simple, y en la medida de lo posible con oposición. En la literatura científica, existen estudios relacionados con las variables pedagógicas analizadas en diferentes modalidades deportivas, como es el caso del baloncesto (Cañadas, Ibáñez et al., 2013; Cañadas, et al., 2015; Cañadas, Rodríguez et al., 2013), y en balonmano (Dallegrave et al., 2018; García-Ceberino et al., 2018) utilizando la herramienta SIATE. Concretamente, en fútbol-base existe un único trabajo previo que analiza las tareas de entrenamiento con el objetivo de conocer las asociaciones entre los medios de iniciación al entrenamiento y las diferentes variables pedagógicas que las definen (Gómez-Carmona et al., 2018). Estos trabajos ponen de manifiesto la importancia de conocer y exponer la realidad de los procesos de entrenamiento y competición con la finalidad de incrementar las posibilidades de rendimiento deportivo (Lago, 2008), y la formación de los deportistas (Ibáñez et al., 2016) en categoría base.

En relación al segundo objetivo, las diferencias existentes en el diseño de tareas de entrenamiento entre los mesociclos analizados se centran en las variables POR y LJ. Sin embargo, el TMe no está influenciado por las variables Situaciones de juego (SJ), Tipo de contenido (CONT-G), Medio de iniciación al entrenamiento (MIE), y Nivel de oposición (NO).

Respecto a la Presencia de portero (POR), los resultados muestran que hay diferencias en el diseño de las tareas de entrenamiento entre los mesociclos analizados. Existe un mayor número de tareas de entrenamiento en el mesociclo VI con portero, y en el mesociclo VII sin portero. Esto es debido a que en el mesociclo VI se llevan a cabo tareas específicas para el desarrollo del portero, las cuales no son realizadas en el mesociclo VII. Esto lleva a pensar que existe un amplio peso de la formación o de la experiencia del técnico deportivo, que determinan la especificidad de las tareas de entrenamiento en función del puesto específico (Cañadas,
Ibáñez, García-Rubio et al., 2011). La presencia del portero en las tareas de entrenamiento es muy importante en los deportes de invasión (Ibáñez et al., 2016), pero una gran cantidad de la tareas utilizadas para el desarrollo de las capacidades técnico-tácticas en el fútbol son los juegos reducidos, donde muchas de sus disposiciones son realizadas sin portero, teniendo esto una influencia directa en aspectos cinemáticos (Gómez-Carmona, Gamonales, Pino-Ortega e Ibáñez, 2018; Reche-Soto, Cardona-Nieto, Díaz-Suárez, Gómez-Carmona y Pino-Ortega, 2019) y tácticos (Reche-Soto, Cardona-Nieto, Díaz-Suárez, Gómez-Carmona y Pino-Ortega, 2018). Este aspecto también ha sido estudiado en otras modalidades deportivas como el balonmano, encontrando que la oposición defensiva o del portero es relevante en el lanzamiento (RivillaGarcía, Navarro \& Sampedro, 2011). Por lo tanto, en el diseño de las tareas de entrenamiento con o sin presencia de portero juega un papel fundamental la formación del técnico y la metodología de enseñanza empleada, pues tiene una influencia directa en la planificación de los contenidos a seguir.

Por otro lado, existen diferencias en el diseño de tareas de entrenamiento entre los mesociclos analizados en relación a la variable Línea de juego / grupo de jugadores $(L J)$. Los resultados muestran que existe un mayor número de casos de tareas de entrenamiento específicas para portero y lateral durante el mesociclo VI, y en el mesociclo VII existe un mayor número de tareas de entrenamiento de equipo. Estos datos son diferentes a los existentes en la literatura científica relacionada con SIATE y la variable línea de juego (Cañadas et al., 2012; Dallegrave et al., 2018; Mancha-Triguero et al., 2018), como consecuencia del contexto deportivo donde se desarrollan estas investigaciones. Sin embargo, los resultados están en consonancia con los descritos por los autores Gómez-Carmona et al. (2018). La tendencia en el fútbol-base es progresar en el diseño de las tareas de entrenamiento, no ocurriendo lo mismo en el caso de los técnicos inexpertos (Cañadas et al., 2013). Por tanto, es necesario ampliar la información en otras modalidades deportivas, con más perfiles de entrenadores y en más categorías de formación, para poder determinar el perfil específico en el diseño de las tareas de entrenamiento.

Las variables que no muestran diferencias significativas son Situaciones de juego (SJ), Tipo de contenido (CONT$G)$, Medio de iniciación al entrenamiento (MIE), y Nivel de oposición (NO).

En relación a las Situaciones de juego (SJ), no existen diferencias entre ambos mesociclos. El técnico deportivo diseñó principalmente situaciones de juego de $8 \times 7$ para el mesociclo VI, y acciones combinadas para el mesociclo VII. Estas tareas de entrenamiento poseen bastantes exigencias cinemáticas y fisiológicas semejantes al juego real (Casamichana, Castellano \& Castagna, 2012), y mejoran la toma de decisiones (Davids, Araújo, Correia \& Vilar, 2013). Por esto, en los resultados no se observan diferencias, pero si en la evolución de las situaciones de juego a lo largo de los dos mesociclos. Sería conveniente analizar un número mayor de mesociclos para comprobar la progresión existente en las tareas de entrenamiento diseñada para el fútbol-base, puesto que está en sintonía y corroboran lo citado por Cañadas et al., (2013) y Cañadas, Ibáñez, García-Rubio et al., (2011), donde se encuentra una progresión en la situaciones de 
juego en relación al nivel de sesión y al periodo de la temporada.

No se identifican asociaciones entre la Fase de juego (FJ) y el Tipo de mesociclo (TMe), lo que demuestra que no existen diferencias en el diseño de tareas entre los dos mesociclos analizados. En ambos mesociclos, el técnico deportivo diseña tareas de entrenamiento mixtas, en consonancia con el contexto deportivo. El fútbol-base se caracteriza por continuos cambios técnico-tácticos entre las fases de juego de defensa y ataque (Gómez-Carmona et al., 2018). Las tareas diseñadas permanecen estables, puesto que el técnico deportivo parte de asumir la realidad de la competición (Casamichana et al., 2012).

Con respecto al Tipo de contenido (CONT-G), no existen diferencias en el diseño de tareas de entrenamiento entre los distintos mesociclos. En ambos mesociclos, predominan los contenidos CTTCA, Calentamiento y Preparación físi$c a$. Estos datos son totalmente diferentes a los existentes en el fútbol, tanto en categorías de formación como en fútbol profesional, centrado en contenidos técnicos (Saad \& Nascimento, 2007), o preparación física (Partington \& Cushion, 2013). Además, es diferente a los estudios existentes con SIATE en baloncesto (Cañadas \& Ibáñez, 2010; Cañadas et al., 2015), y balonmano (Dallegrave et al., 2018; GarcíaCeberino et al., 2018). Sería conveniente ampliar la muestra del estudio, así como analizar las tendencias de los técnicos deportivos en el fútbol-base y comprobar que medios utilizan para desarrollar los objetivos propuestos en sus sesiones de entrenamiento como en la investigación de Gamonales et al., (2019).

En relación a la variable Medio de iniciación al entrenamiento (MIE), no se aprecian diferencias. En ambos mesociclos, permanece estable los medios de iniciación al entrenamiento. El técnico deportivo diseña tareas de entrenamiento en las cuales utiliza el juego complejo específico y ejercicios de aplicación simple para desarrollar los objetivos deportivos. Estos datos están próximos a los expuestos previamente por Gómez-Carmona et al., (2018), donde el entrenador diseña tareas de entrenamiento utilizando el juego complejo específico y el pre-deporte/ deporte adaptado como medios para alcanzar las finalidades propuestas. Esta variación puede ser consecuencia del número de mesociclos analizados, así como del momento de la temporada.

Finalmente, no existen diferencias entre el Nivel de oposición (NO) y el Tipo de mesociclo (TMe). Sin embargo, los resultados descriptivos muestran que durante los dos mesociclos el técnico deportivo diseña tareas con oposición. Estos datos están en sintonía con lo apuntado por Gómez-Carmona et al., (2018), y señalan que las tareas diseñadas deben fomentar la toma de decisiones de los jugadores a nivel técnico-táctico mediante el aprendizaje de conductas sobre el desarrollo individual a través de los gestos técnicos específicos, para conseguir una mayor riqueza técnico-táctica (Gray \& Sproule, 2011) y simular los requerimientos de la competición (Torres-Ronda, Ric, Llabres-Torres, De las Heras, \& Del Alcazar, 2016). Por tanto, este tipo de estudios supone un importante punto de partida, pues permite conocer y analizar las tendencias en el diseño de tareas de entrenamiento en el fútbol-base de los técnicos, así como conocer la realidad deportiva en el proceso de formación.

\section{Limitaciones}

Aunque el estudio describe el tipo de tareas diseñadas por un entrenador de fútbol-base sub-18 y analiza la influencia del Tipo de Mesociclo (TMe) en las variables pedagógicas, diferentes limitaciones deben ser consideradas. En primer lugar, el estudio fue realizado en un único club de fútbol de categoría juvenil, donde un entrenador con unas características específicas fue el encargado de diseñar las tareas. Por tanto, los resultados no son generalizables a otras categorías e incluso a otros entrenadores que presenten diferentes características y formación que el evaluado. Aun así, este es un trabajo pionero que permite aumentar el conocimiento del análisis de las tareas de entrenamiento en el fútbol de formación. Investigaciones futuras deberían profundizar en la influencia de las variables contextuales sobre las tareas de entrenamiento, entre las que destacan la modalidad deportiva, el nivel competitivo, así como la diferencia entre categorías de fútbol-base. Además de la manipulación de variables contextuales, sería de interés aumentar los clubs participantes para analizar la influencia de las características del entrenador de forma específica entre equipos de una misma categoría.

\section{Conclusiones}

El entrenador de fútbol-base analizado en esta investigación utiliza principalmente las siguientes variables pedagógicas: a) Situaciones de Juego: 8x7 y combinado; b) Presencia de Portero: Sin presencia de portero; c) Fase de Juego: Mixta, combinación de las fases de ataque y defensa dentro de la misma tarea; d) Tipo de Contenido: Conductas técnico-tácticas colectivas de ataque; e) Medio de iniciación al entrenamiento: juego complejo específico; f) Línea de juego / grupo de jugadores: equipo; y, por último, g) Nivel de Oposición: Con oposición, con la finalidad de desarrollar los contenidos técnico-tácticos, físicos y psicológicos de los jugadores juveniles.

En relación con las diferencias en el diseño de tareas, las principales asociaciones se encuentran en las variables de estudio POR y $L J$, encontrando un grado de asociación bajo. Por ello, es importante diseñar entrenamientos donde se trabajen las diferentes líneas de juego, así como la presencia de portero en las tareas.

\section{Referencias}

Alarcón, F., Cárdenas, D., Miranda, M.T., Ureña, M., \& Pilar, M.I. (2010). La metodología de enseñanza en los deportes de equipo. Revista de Investigación en Educación, 7,91-103.(

Ato, M., López, J.J., \& Benavente, A. (2013). Un sistema de clasificación de los diseños de investigación en psicología. Anales de Psicología, 29(3), 1038-1059.

Bastida-Castillo, A., Gómez-Carmona, C. D., De la CruzSánchez, D., Reche-Royo, X., Ibáñez, S. J., \& Pino Ortega, J. (2019). Accuracy and Inter-Unit Reliability of UltraWide-Band Tracking System in Indoor Exercise. Applied Sciences, 9(5), 939.

Bastida-Castillo, A., Gómez-Carmona, C. D., De la Cruz 
Sánchez, E., \& Pino-Ortega, J. (2019). Comparing accuracy between global positioning systems and ultra-widebandbased position tracking systems used for tactical analyses in soccer. European Journal of Sport Science, Pendiente de Publicación.

Cañadas, M., \& Ibáñez, S.J. (2010). La planificación de los contenidos de entrenamiento de baloncesto con equipos de iniciación. E-Balonmano: Revista de Ciencias del Deporte, 6(1), 49-65.

Cañadas, M., Ibáñez, S.J., \& Leite, N. (2015). A novice coach’s planning of the technical and tactical content of youth basketball training: A case study. International Journal of Performance Analysis in Sport, 15(2), 572-587. DOI: http://doi.org/10.1080/24748668.2015.11868815

Cañadas, M., Ibáñez, S.J., Feu, S., García-Rubio, J., \& Parejo, I. (2011). Análisis de los medios de entrenamiento en un equipo minibasket y la influencia de un programa formativo para el entrenador. Un estudio de caso. ÁGORA para la Educación Física y el Deporte, 13(3), 363-382.

Cañadas, M., Ibáñez, S.J., García-Rubio, J., \& Sáez, J. (2010). Influencia de un programa formativo en el empleo de los medios de entrenamiento en equipos de baloncesto en período de formación. Cultura, Ciencia y Deporte, 13(5), 95.(

Cañadas, M., Ibáñez, S.J., García-Rubio, J., Parejo, I., \& Feu, S. (2011). Las situaciones de juego en el entrenamiento de baloncesto en categoría base. Revista Internacional de Medicina y Ciencias de la Actividad Física y el Deporte, 13(45), 41-54.

Cañadas, M., Ibáñez, S.J., García-Rubio, J., Parejo, I., \& Feu, S. (2012). Estudio de las fases de juego a través del análisis del entrenamiento deportivo en categoría minibasket. Cuadernos de Psicología del Deporte, 12(2), 73-82.(

Cañadas, M., Ibáñez, S.J., García-Rubio, J., Parejo, I., \& Feu, S. (2013). Game situations in youth basketball practices. Revista Internacional de Medicina y Ciencias de la Actividad Física y del Deporte, 13(49), 41-54.

Cañadas, M., Parejo, I., Ibáñez, S.J. García-Rubio, J., \& Feu, S. (2009). Relación entre las variables pedagógicas de los entrenadores de baloncesto que trabajan en etapas de formación. Habilidades Motrices: Revista de Ciencias de la Actividad Física y del Deporte, 32, 44-52.

Cañadas, M., Rodríguez, G., Feu, S., Parejo, I., \& García-Rubio, J. (2013). Relathionship between pedagogical content knowledge and coaching methods. Revista de Psicología del Deporte, 22(1), 183-186.

Casamichana, D., Castellano, J., \& Castagna, C. (2012). Comparing the physical demands of friendly matches and small-sided games in semiprofessional soccer players. The Journal of Strength \& Conditioning Research, 26(3), 837-843. http://doi.org/10.1519/ JSC.0b013e31822a61cf

Clemente, F.M., Martins, F.M., \& Mendes, R.S. (2015). How coaches use their knowledge to develop small-sided soccer games: a case study. South African Journal for Research in Sport, Physical Education and Recreation, $37(1), 1-11$.

Coque, I. (2008). Valoración subjetiva de la carga del entrenamiento técnico-táctico. Una aplicación práctica (I). Clinic, (81), 39-43.
Crewson, P. (2006). Applied statistics handbook. AcaStat Software, Leesburg.

Dallegrave, E.J., Mendes, J. C., \& Vieira do Nascimento, J. (2018). Estrutura organizativa do processo de treinamento técnico-tático nas categorias de formação no handebol feminino. E-balonmano.com: Revista de Ciencias del Deporte 14(2), 65-70.

Davids, K., Araújo, D., Correia, V., \& Vilar, L. (2013). How small-sided and conditioned games enhance acquisition of movement and decision-making skills. Exercise and Sport Sciences Reviews, 41(3), 154-161. http://doi.org/ 10.1097/JES.0b013e318292f3ec

De la Fuente-Fernández, S. (2016). Aplicaciones de la Chicuadrado: tablas de contingencias. Homogeneidad. Dependencia e independencia. Madrid: Universidad Autónoma de Madrid.

Del Campo, J., Álvarez, J., \& Lorenzo, A. (2008). La percepción del esfuerzo: concepto, características y aplicación al control del entrenamiento en baloncesto. En: Terrados, N., y Calleja, J. (eds.). Fisiología, Entrenamiento y Medicina del Baloncesto. $1^{\text {a }}$ Edición (pp. 121-134). Badalona: Paidotribo.

Field, A. (2009). Discovering statistics using SPSS (3a ed.). London: Sage Publications Ltd.

Gamonales, J.M., Gómez-Carmona, C.D., León, K., GarcíaSantos, D., Gamero-Portillo, M.G., \& Muñoz-Jiménez, J. (2019). Análisis de las tareas de Entrenamiento en fútbolbase: diferencias entre dos meses durante el period competitive en la categoría sub-19. Sportis: Revista Técnico-Científica del Deporte Escolar, Educación Física y Psicomotricidad, 5(1), 30-52. http://doi.org/10.17979/ sportis.2019.5.1.3469

Gamonales, J.M., Muñoz-Jiménez, J., León-Guzmán, K., \& Ibáñez, S.J. (2018). Entrenamiento y confiabilidad entre observadores en el análisis del fútbol para ciegos. Retos: Nuevas tendencias en Educación Física, Deporte y Recreación, 34(2), 155-161.

García-Ceberino, J.M., Gamero, M.G., González-Espinosa, S., García- Rubio, J., \& Feu, S. (2018). Estudio de la carga externa de las tareas para la enseñanza del balonmano en función del género de los profesores en formación. Ebalonmano.com: Revista de Ciencias del Deporte, 14(1), 45-54.

Gómez-Carmona, C.D., García-Rubio, J., Muñoz-Jiménez, J., \& Gamonales, J.M. (2018). Relación entre el medio de iniciación al entrenamiento y las variables pedagógicas que definen las tareas en el fútbol. TRANCES: Revista de Transmisión del Conocimiento Educativo y de la Salud, 10(1), 401-420.

Gómez-Carmona, C., Gamonales, J., Pino-Ortega, J., \& Ibáñez, S. (2018). Comparative Analysis of Load Profile between Small-Sided Games and Official Matches in Youth Soccer Players. Sports, 6(4), 173.

González-Espinosa, S., Ibáñez, S.J., \& Feu, S. (2017). Diseño de dos programas de enseñanza del baloncesto basados en métodos de enseñanza-aprendizaje. $E$ balonmano.com: Revista de Ciencias del Deporte, 13(2), 131-152.

González-Espinosa, S., Ibáñez, S. J., Feu, S., \& Galatti, L. R. (2017). Programas de intervención para la enseñanza de- 
portiva en el contexto escolar, PETB y PEAB: Estudio preliminar. Retos: Nuevas tendencias en Educación Física, Deporte y Recreación, (31), 107-113.

Gracia, F., García-Rubio, J., Cañadas, M., \& Ibáñez, S.J. (2014). Diferencias en la frecuencia cardíaca en situaciones de juego modificadas en baloncesto de formación. Ebalonmano.com: Revista de Ciencias del Deporte, 10(1), 23-30.

Gray, S., \& Sproule, J. (2011). Developing pupils’ performance in team invasion games. Physical Education and Sport Pedagogy, 16(1), 15-32.

IbaìnPez, S. J. (2002). Los contenidos de ensenPanza del baloncesto en las categoriìas de formacioìn. In S. J. IbaìnPez \& M. M. Maciìas (Eds.), Nuevos horizontes para o treino do baisquetbol (pp. 111-136). Cruz Quebrada: Edic’oDes FMH, Facultade de Motricidade Humana.

Ibáñez, S.J., Feu, S., \& Cañadas, M. (2016). Sistema integral para el análisis de las tareas de entrenamiento, SIATE, en deportes de invasión. E-balonmano.com: Revista de Ciencias del Deporte, 12(1), 3-30.

Kirk, D., \& MacPhail, A. (2002). Teaching games for understanding and situated Learning: rethinking the bunker-thorpe model. Journal of Teaching in Physical Education, 21(2), 177-192.(

Lago, C. (2008). El análisis del rendimiento en el fútbol. Estado actual y perspectivas de futuro en la investigación. En J. Castellano (Eds.), Fútbol e innovación (pp. 89-103). Sevilla: Wanceulen.

Luteberget, L. S., Spencer, M., \& Gilgien, M. (2018). Validity of the Catapult ClearSky T6 local positioning system for team sports specific drills, in indoor conditions. Frontiers in Physiology, 9, 115.

Malone, J. J., Lovell, R., Varley, M. C., \& Coutts, A. J. (2017). Unpacking the black box: applications and considerations for using GPS devices in sport. International Journal of Sports Physiology and Performance, 12(Suppl 2), S218.

Mancha-Triguero, D., García-Ceberino, J.M., Antúnez, A., \& García-Rubio, J. (2018). ¿Afecta la fase de juego al diseño de las tareas de un equipo de baloncesto de formación?. SPORT-TK: Revista Euroamericana de Ciencias del Deporte, 7(2), 27-36.

Newell, J., Aitchison, T., \& Grant, S. (2014). Statistics for sports and exercise science: a practical approach. New York: Routledge.

Partington, M., \& Cushion, C. (2013). An investigation of the practice activities and coaching behaviors of professional top level youth soccer coaches. Scandinavian Journal of Medicine \& Science in Sports, 23(3), 374- 382. (

Randolph, J.J. (2005). Free-Marginal Multirater Kappa (Multirater Kfree): An Alternative to Fleiss ì Fixed-Marginal Multirater Kappa. Joensuu Learning and Instruction Symposium 2005, University of the Joensuu, Finland.

Reche-Soto, P., Cardona-Nieto, D., Díaz-Suárez, A., GómezCarmona, C. D., \& Pino-Ortega, J. (2018). Demandas tácticas de juegos reducidos en fútbol: influencia de la tecnología utilizada. Revista Internacional de Medicina y Ciencias de la Actividad Física y el Deporte, Pendiente de Publicación. (

Reche-Soto, P., Cardona-Nieto, D., Díaz-Suárez, A., Gómez-
Carmona, C. D., \& Pino-Ortega, J. (2019). Análisis de las demandas físicas durante juegos reducidos en fútbol semi-profesional en función del objetivo y la tecnología de seguimiento utilizada. E-balonmano. com: Revista de Ciencias del Deporte, 15(1), 23-36.

Reina, M., Mancha-Triguero, D., Feu, S., \& Ibáñez, S.J. (2017). ¿Se entrena como se compite? Análisis de la carga en baloncesto femenino. Revista de Psicología del Deporte, 26(1), 9-13.

Rivilla-García, J., Navarro, F., \& Sampedro, J. (2011). Influencia de la oposición del portero en la precisión del lanzamiento en jugadores sénior y sub-18 de balonmano. Ebalonmano.com: Revista de Ciencias del Deporte, 7(1), 17-25.

Saad, M.A., \& Nascimento, J.V. (2007). Estruturação das sessões de treinamento técnico-tático nos escalões de formação do futsal. Revista Portuguesa de Ciências do Desporto, 7(1), 21-28. (

Sáenz-López, P., Feu, S., \& Ibáñez, S.J. (2006). Estudio de la participación de los jugadores españoles de baloncesto en las distintas categorías de la selección nacional. Apunts: Educación Física y Deporte, 85, 36-45. (

Sánchez, M. (2007). El acondicionamiento físico en baloncesto. Apunts: Educación Física y Deporte 42, 99-107.

Silva, A., Sánchez-Bañuelos, F., Garganta, J., \& Anguera, M. T. (2005). Patrones de juego en el fútbol de alto rendimiento. Análisis secuencial del proceso ofensivo en el campeonato del mundo Corea-Japón 2002. Cultura, Ciencia y Deporte, 1, 65-72.

Torres-Ronda, L., Ric, A., Llabres-Torres, I., De las Heras, B., y Del Alcazar, X.S. (2016). Position-dependent cardiovascular response and time-motion analysis during training drills and friendly matches in elite male basketball player. The Journal of Strength \& Conditioning Research, 30(1), 60-70. http://doi.org/10.1519/ JSC.0000000000001043

Villarejo, D., Ortega, E., Gómez-Ruano, M.A., \& Palao, J. (2014). Design, validation and reliability of an observational instrument for ball possessions in rugby unión. International Journal of Performance Analysis, 14(3), 896-908. http://doi.org/10.1080/24748668.2014.11868771

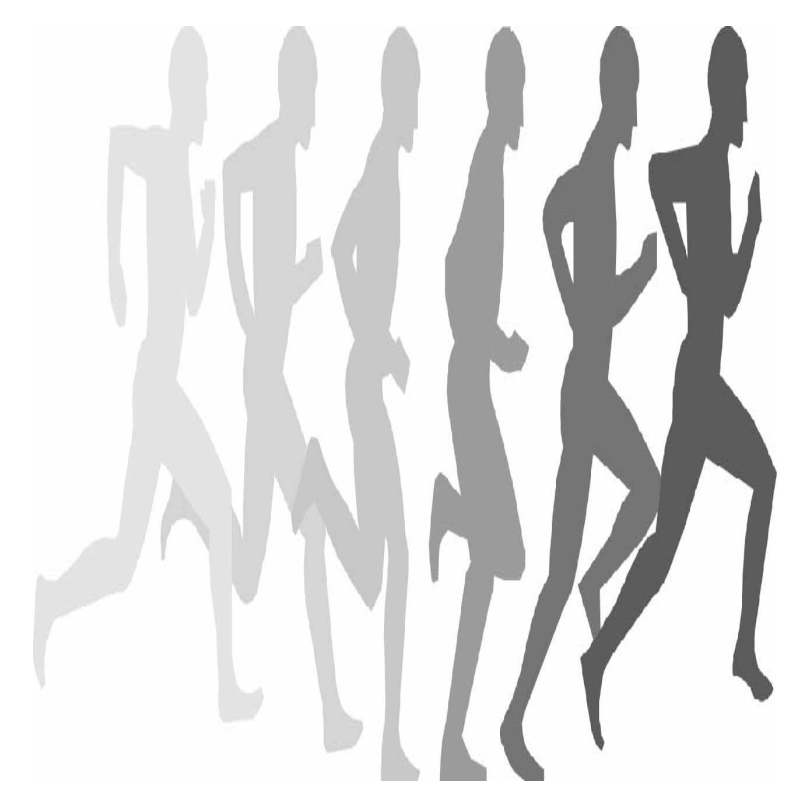

